\title{
Novel real-time closed-loop device linearization via predictive pre-distortion
}

\author{
Walid Ahmed ${ }^{1}$, Ajit Reddy ${ }^{2, *}$ \\ ${ }^{1}$ Verizon Wireless, New Jersey, USA \\ ${ }^{2}$ Alcatel-Lucent, New Jersey, USA
}

Email address:

walidmail@yahoo.com (W.Ahmed), ajitk_reddy@yahoo.com (A. Reddy)

\section{To cite this article:}

Walid Ahmed, Ajit Reddy. Novel Real-Time Closed-Loop Device Linearization via Predictive Pre-Distortion. Science Journal of Circuits, Systems and Signal Processing. Vol. 3, No. 3, 2014, pp. 14-25. doi: 10.11648/j.cssp.20140303.11

\begin{abstract}
In this paper, a novel real-time closed-loop device linearization technique has presented. In this paper the focus application is on $\mathrm{AM} / \mathrm{AM}$ and $\mathrm{AM} / \mathrm{PM}$ linearization of power amplifiers (PA) and/or radio transmitters. In such an application, the novel approach performs on-the-fly measurement-and-prediction of the nonlinear characteristics of the PA, stores such nonlinear characteristics and calculates their inverse functions in order to pre-distort the base-band amplitude and phase signals modulating the PA such that the combination, or the resultant, of the pre-distorter and the PA leads to a linear behavior at the output of the PA. The predictive nature of the presented approach overcomes the inevitable delay between the time a measurement is collected through the feedback loop and the time it has taken place at the output of the forward loop, which is a must-have delay encountered in any natural causal system. Such a delay results in imperfect on-the-fly pre-distortion of the output signal due to the mismatch between the applied pre-distortion, which has been based on a past measurement, and the actual effect of the non-linearity at the time the signal is produced [5]. In addition, this novel approach promises the advantage of operating a highly non-linear (compressed) PA - hence, highly efficient - with minimal factory pre-calibration. We present a model of our predictor based approach and evaluate its performance for a GSM/EDGE/UMTS a cellular transmitter scenario, where the performance requirements on the transmitted signal are stringent and distortion due to non-linearity must be minimized. The key performance metrics we evaluate have mostly been based on the GSM/EDGE/UMTS requirements, such as the Error Vector Magnitude (EVM), Switching Transients (ST), and Adjacent Channel Power Ratio (ACPR), transmit time mask, modulation spectrum and power-added efficiency (PAE). Although the focus of the numerical results in this paper is on the GSM/EDGE/UMTS application, the novel approach discussed is applicable to any TDMA or TDD system where switching transients and spectral performance is tightly controlled.
\end{abstract}

Keywords: Power Amplifier, Non-Linearity, Pre-Distortion, Real-Time Calibration, Real-Time Prediction

\section{Introduction}

With the growing rate of cell phone users and the demand for higher data rates, the requirements on information throughput and reliability are also increasing. These requirements have led to the development of new technologies that aim at satisfying such challenging requirements. With the advancement in digital technologies, it is now possible to increase the capacity several times higher than what the analog systems could deliver. Some of the basic techniques employed to achieve this are multiple access, channel coding and modulation. For cellular systems, the capacity can also be increased with more flexible cellplanning and smaller cells. Until recently, a large portion of the land mobile radio systems were based on the GSM standard, for example, which uses the GMSK constantenvelope modulation technique. The advantage with constant-envelope modulation is that the transmitter's power amplifier (PA) - which is especially important for the mobile equipment because it is naturally one of the most "power" consuming parts - does not have to be linear, hence can be made more efficient. The major drawback with such modulation techniques is the inefficient use of the allocated radio spectrum. On the other hand, spectrally efficient systems use modulation techniques that have both a varying phase and a varying envelope as in the EDGE system, which uses 8-PSK modulation. If we apply an M-ary signal with a varying envelope to a power efficient but rather non-linear 
amplifier, the radio communication system will cause a number of impairments to the transmitted signal: First, the non-linear characteristics of the power amplifier will cause time-domain signal distortion that results in re-growth of the transmitted signal spectrum, which in-turn leads to spectral expansion, or leakage, into adjacent channels causing interference for other users. Second, the time-domain signal distortion makes it difficult for the receiver to correctly demodulate and decode the information. Therefore, such distortion due to a non-linear power amplifier must be corrected, i.e., linearized. However, ultimately the intent is to linearize the PA not by designing linear inefficient power amplifiers, but rather by pre-distortion of non-linear (thus efficient) power amplifiers.

In this paper ${ }^{1}$, we present a novel real-time closed-loop device linearization technique that has originally been proposed by Ahmed and Li [1]. The focus application is on $\mathrm{AM} / \mathrm{AM}$ and $\mathrm{AM} / \mathrm{PM}$ linearization of power amplifiers and/or radio transmitters wherein $\mathrm{AM}$ is the Amplitude Modulation and PM is the Phase Modulation. In such an application, this novel approach performs on-the-fly measurement-and-prediction of the nonlinear characteristics of the PA, stores such non-linear characteristics and calculates their inverse functions in order to pre-distort the base-band amplitude and phase signals modulating the PA such that the combination, or the resultant, of the predistorter and the PA leads to a linear behavior at the output of the PA. The predictive nature of this approach overcomes the inevitable delay between the time a measurement is collected through the feedback loop and the time it has taken place at the output of the forward loop, which is a must-have delay encountered in any natural causal system. Such a delay results in imperfect on-the-fly pre-distortion of the output signal due to the mismatch between the applied pre-distortion, which has been based on a past measurement, and the actual effect of the non-linearity at the time the signal is produced [5].

The novel approach, thus, promises the advantage of operating a highly non-linear (compressed) PA - hence, highly efficient - with minimal factory pre-calibration. We present a model of our predictor based approach and evaluate its performance for a GSM/EDGE cellular transmitter scenario, where the performance requirements on the transmitted signal are stringent and distortion due to nonlinearity must be minimized, especially during the power ramp-up period of an EDGE/GSM burst, where switching transients are of great concern. Therefore, linearity of the transmitter during ramp-up is imperative.

It should be emphasized that the predictive method presented is very different from the correction techniques generally found in literature which are used to correct for the non-linearity of power amplifiers. A key advantage of this novel technique is that the approach is of reduced complexity and does not involve the use of complex matrix/LUT

\footnotetext{
${ }^{1}$ This work has been performed while the authors have been with M/ACOM Inc.
}

computations that are typically required for the inversion (pre-distortion) of the PA non-linearity characteristics. In addition, this approach is able to adapt and correct the nonlinearity in real-time (e.g., from burst to burst GSM/EDGE systems). Our approach is also able to do this with a time complexity of $O(N)$. Hence, our method, as will be discussed in the following sections, demonstrates not only the novelty but also simplicity from an implementation perspective.

To further clarify the motive behind the proposed approach, we present the following argument which is motivated by the GSM/EDGE case study/example. When the PA AM/AM and $\mathrm{AM} / \mathrm{PM}$ are not known to the transmitter beforehand (e.g., via factory calibration), then we are faced with a chickenand-egg problem on start-up of an EDGE/GSM burst that can be explained in the following set of bullet points:

- Every new burst can, in principle, have a new carrier frequency (due to EDGE/GSM frequency hopping) and a new average power level requirement (based on the network requirement assigned to that burst). In addition and due to varying environmental conditions surrounding the mobile handset, it is expected to also suffer variable electro-magnetic (EM) "loading" effects, hence, encountering variable Voltage Standing-Wave Ratio (VSWR).

- The use of a highly efficient PA implies severe nonlinearity. Hence, PA characteristics need to be corrected for, or linearized, in baseband, which involves predistortion of the baseband signal prior to modulating the PA. Otherwise, acceptable spectral and waveform quality performance can not be achieved for the transmitted signal.

- The severe PA non-linearity is also expected to change versus many parameters, such as temperature, supply voltage, PA power level setting (PA bias), PA load (VSWR), PA part-to-part tolerance (variation) and aging.

- Open-loop (factory) calibration of such a non-linear PA behavior is difficult since it is not possible to store such a large number of PA correction tables versus so many permutations of operating conditions (or parameters).

- Such a constraint implies that the transmitter must employ some sort of on-the-fly calibration mechanism that monitors the PA output versus its input to characterize the PA non-linearity on-the-go. However, on-chip non-real-time closed-loop solutions, e.g., calibrate the PA before every call or when turning the phone on and use the generated tables for the entire transmission duration, may not work if the PA is expected to change its AMAM/AMPM behavior during transmission due to, for example, load and VSWR changes, or carrier frequency changes due to frequency hopping.

- Hence, frequent calibration is needed, which implies the need for an adaptive closed-loop solution. Two challenges come with such an approach:

- When to calibrate? Standards usually do not allocate off-air time for such an operation to be done! 
○ How to maintain signal quality while calibrating and transmitting at the same time?

- In EDGE/GSM, for example, the switching-transient requirements are very stringent. It is not possible to let the PA run free of correction (i.e., without linearization) at any point in time. It is also not possible to have inaccurate correction tables applied to the PA. Moreover, a high-efficient PA will have a high level of non-linearity that will change from one EDGE/GSM burst to the next due to (at least) load/VSWR changes. Hence, pre-stored (or pre-loaded) correction tables will not suffice.

- How and when do we calibrate the highly-non-linear PA in an EDGE/GSM application during EVERY BURST is discussed. Calibration of the PA is done during the EDGE/GSM burst ramp-up. In order to do that, the power ramp-up profile is designed in a special way to ensure all amplitude levels are covered, yet a smooth rising ramp is achieved in order not to violate the tough EDGE/GSM switching-transients requirements. Details of such a consideration are beyond the scope of this paper. As mentioned earlier, a simple, novel, robust and arbitrarily tunable ramp-up design technique has been proposed by Ahmed [4].

Regarding to the discussion as to how the calibration is done, is the focus of this paper. The strategy adopted to solve the chicken-and-egg problem is further presented:

- A major challenge in calibrating on the ramp is that while collecting the PA response data, the data is also being transmitted. Hence, the correction data must be available to ensure quality transmission, but clearly, during this time the correction is not yet done since the calibration process is on.

- Moreover, the attempt at performing such an on-the-fly correction/linearization of the PA while ramping up will suffer from memory effects, that is, the collected measurements of the PA AM/AM and AM/PM nonlinearity lag behind the current bias point of the PA due to the inevitable forward and feedback loop path delays Hence, resulting-in inaccurate correction (or inversion of the PA non-linearity), which clearly leads to unacceptable performance that fails the stringent requirements of the EDGE/GSM specifications.

- Accordingly, one must use a mechanism that can break into the future in some sense and predict what the next non-linearity point on the PA curve would look like based on previous on-the-fly measurements.

The key performance metrics evaluated have mostly been based on the GSM/EDGE requirements, such as the Error Vector Magnitude (EVM), Switching Transients (ST), Adjacent-Channel Power Ratio (ACPR), Transmit TimeMask (TTM), Modulation Spectrum (MS), and Power-Added Efficiency (PAE). It should be noted that the power amplifiers for wider bandwidths do exhibit memory effects, but for the bandwidths discussed in this paper for GSM/EDGE and UMTS the memory effects exhibited by the power amplifiers is not a problem.
Finally, we would like to repeat the emphasis on the fact that the key advantage of our proposed technique over the linearization techniques discussed in literature (e.g., [1015]) is that it is capable, right from the transmission of the first data frame, of achieving on-the-fly clean transmission (i.e., without any spectral re-growth or signal regeneration seen in the output spectrum, including the ramp-up and ramp-down periods (e.g., in a TDMA, or TDD, system)) without the need for any a-priori knowledge of the PA nonlinearity, i.e., unlike other linearization methods that must use pre-loaded pre-measured pre-distortion LUTs in order to achieve no spectral re-growth while in real-time transmission.

This paper is organized as follows. The next section introduces our novel predictive linearization scheme. Then, Section III provides a simplified mathematical framework for the performance evaluation of our novel approach. Section IV provides an introduction to the performance metrics we adopted in order to qualify the performance of our novel approach, while Section V provides detailed performance results that have been based on extensive modeling and simulations of our transmitter and power amplifier line-up, which has been developed at M/A-COM, Tyco Electronics. Finally, Section VI provides the conclusions for this paper.

\section{Predictor Model}

Current PA linearization techniques generally follow one of two approaches. The first is to use pre-measured (e.g., factory calibrated) correction tables which can be considerably inaccurate/mismatched if the PA characteristics change over temperature, process and power levels. Such mismatches would result in un-acceptable performance when applied in spectrally-stringent systems such as GSM/EDGE which imposes difficult switching transient requirements. The second approach is to use some sort of on-the-fly correction of the PA. Unfortunately, and as said earlier, existing techniques that attempt at performing on-the-fly correction/linearization of the PA suffer from memory effects. That is, the collected measurements of the PA AM/AM and $\mathrm{AM} / \mathrm{PM}$ non-linearity lag behind the current bias point of the PA due to the inevitable feedback loop path delay (see, for example [5]). Accordingly, resulting in inaccurate correction (or inversion of the PA non-linearity), which in-turn leads to un-acceptable performance that fails the stringent requirements of the GSM/EDGE specifications. As a consequence, one must use a mechanism that can break into the future in some sense and predict what the next nonlinearity point on the PA curve would look like based on previous on-the-fly measurements. Such an approach can be used, for example, while ramping-up the RF power (or waveform) in the beginning of an EDGE burst2. As the

\footnotetext{
2 In this paper, we will provide only a brief introduction to the power ramp-up requirements for GSM/EDGE bursts. Details of the requirements on how to rampup in GSM/EDGE are generally beyond the scope of this paper and are detailed in [2][3]. Also, a novel ramp-up mechanism that meets the stringent GSM/EDGE specs and simultaneously provides a calibration/prediction training waveform has been proposed by Ahmed in [4]
} 
power ramps up to the final level where actual data modulation commences, the transmitter feedback loop collects measurements of the PA and uses them to predict the next linearization value to be applied at the next sample on the ramp-up in order to bias (pre-distort) the PA. This is the gist of our novel predictive linearization approach.

Figure 11 depicts a generic pictorial illustration of the non-linear closed loop prediction technique. As seen in the figure, the non-linear device is placed in a closed-loop that is able to measure 3 the output of the device and feed it back for further "intelligent" signal processing that is based on both the history of the measurements taken at the output of the non-linear device and on comparison with the target signal being supplied at the input of the device.

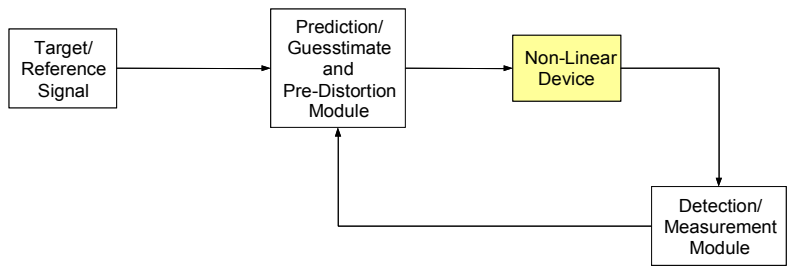

Figure 1. Generic Pictorial Illustration of the Proposed Technique.

The output, or decision, of the intelligent signal processing module (in Figure 1, this module, has been addressed as the "Prediction/Guesstimate" module) is then passed through for further processing required by the system, e.g. clean-up filtering. The output of such a filtering block is then provided to the non-linear device input. This "Prediction/Guesstimate" module is the "heart" of our novel linearization engine. It operates by collecting the measured data points via the feedback path and constructing a "Non-Linear" transfer function4

$$
\mathbf{x}=f^{-1}(\mathbf{y})
$$

where $\mathbf{x}=\left[x_{0}, \ldots, x_{k-1}\right]$ represent the $\mathrm{x}$-axis coordinates (i.e., the signal value at the device input) of the $\mathrm{k}$ data points collected up to the current time instant, and $\mathbf{y}=\left[y_{0}, \ldots, y_{k-1}\right]$ represent the corresponding y-axis coordinates (i.e., the signal value at the device output). Upon construction of the above transfer function, the prediction module estimates the "pre-distorted" value that will be used as the current input value, $x_{k}$, to the non-linear device by "extrapolation" if the target value, $\hat{y}_{k}$, is out of range with respect to the measured data, or by "interpolation" if the target value is within range. Clearly, some error, $e_{k}=y_{k}-\hat{y}_{k}$ is expected, where $y_{k}$ is the actual

3 In the transmitter power amplifier application, the measurement/detection module can be a RF-to-baseband down-converter circuit. For the results in presented in this paper, we have used the novel on-chip differential-phase downconverter technique proposed by Ahmed and Douglas in [6]. This novel technique offers the advantages of completely eliminating phase noise effects on the detected AM/PM distortion and also zooms into only the Tx/PA AMPM distortion by subtracting out the modulating phase signal.

4 Clearly, a linear transfer function becomes a special case of this general mathematical treatment. response of the non-linear device to the input excitation $x_{k}$, but proper averaging and filtering can smooth out most of the noise caused by the numerical error as will be seen in the performance results section.
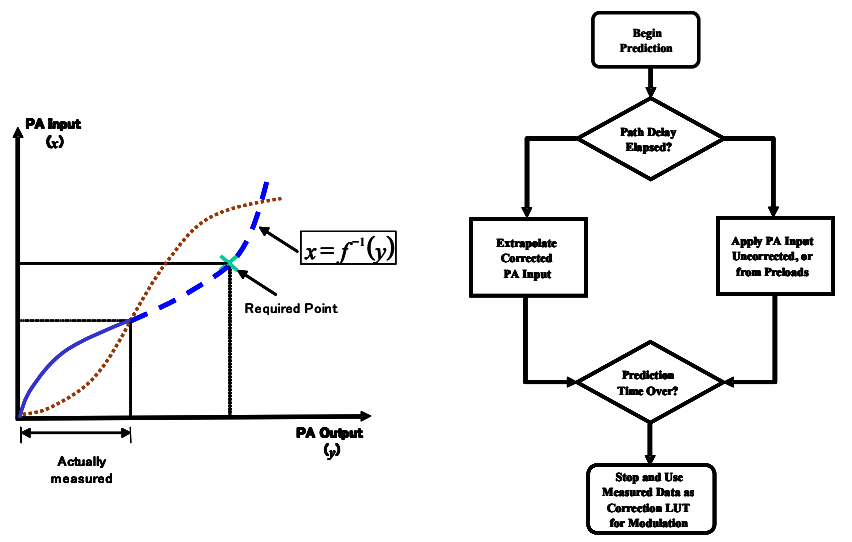

Figure 2. Generic Flow Chart of the Non-Linear Predictive Algorithm.

It should be noted that in some scenarios, it may be expected that a possible "path delay", say L sample periods, exists between the insertion point where the $\mathrm{x}$ values are physically applied and the measurement point where the $y$ values are physically collected. In this case, the first $L$ values of the output measured vector $y$, let them be denoted $y_{-L}, \ldots, y_{-1}$, can either be discarded, or treated in a way that accounts for the fact that they are output responses to inputs that have taken place - in time - prior to the input values $\mathbf{x}=\left[x_{0}, \ldots, x_{k-1}\right]$.

A simplified (and rather generic) flow chart of the predictive algorithm is depicted in Figure 2. The figure assumes that the PA is the non-linear device example. As seen in the flow-chart, the algorithm starts by using either a pre-loaded pre-distortion non-nonlinearity correction characteristics table, or no correction in that initial period where we know the loop delay time-period did not elapse yet, hence the loop output is not really the PA response to the input excitation but rater a response to some pre-ramp-up noise. Once the loop-delay period is elapsed, now the predictive correction begins to take place as explained above, i.e., using (predictive) extrapolation. When the ramp-up (which is also the training period as explained above) is completed the predictive algorithm stops since now the entire non-linearity pre-distortion table has been collected and the system switches to standard LUT pre-distortion non-linearity correction. That is, it pre-distorts the input signal to the PA based on the LUT it has collected during the ramp-up period.

In general, the extrapolation/interpolation algorithm can take on various ways. For example, one may use Linear, Spline, Cubic-Hermite, Polynomial (e.g., quadratic, cubic... etc.), or other mathematical numerical techniques that may best suit the specific case at hand. For example a simplified $1^{\text {st }}$ order extrapolation (piece-wise-linear) is illustrated in Figure 3. 

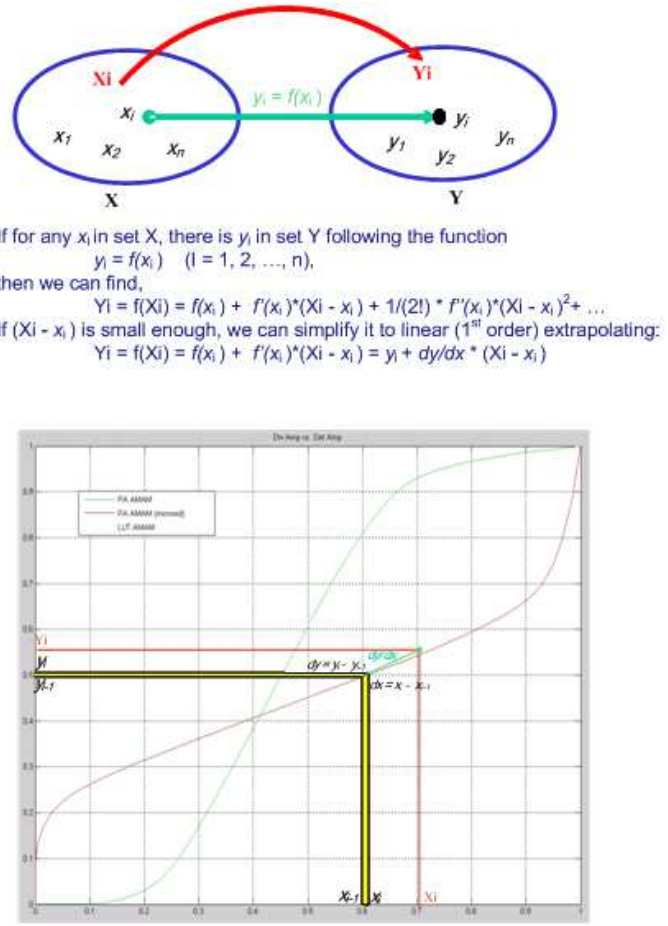

Figure 3. Example of 1st Order Extrapolation.

In addition to the choice of the extrapolation / interpolation method, one may also apply some suitable filtering / averaging (equally or non-equally weighted) to the collected data prior to the extrapolation / interpolation step and/or the estimated pre-distortion values within/after the extrapolation / interpolation step.

\section{Mathematical Analysis}

In this section, we provide a simplified mathematical frame work that aims at shedding some light on the requirement for a satisfactory prediction performance. Without loss of generality, let $x(t)$ denote the desired signal at the output of the non-linear device. Also, let $x(t)$ be a "normalized" linear ramp signal defined as

$$
x(t)=\left\{\begin{array}{cc}
t & 0 \leq t \leq 1 \\
0 & \text { Otherwise }
\end{array}\right.
$$

It should be noted that the "normalized" notion bears the underlying assumption that the non-linear device has an output span that is also from 0 to 1 . In an ideal pre-distortion scenario, i.e., if the transmitter is fully aware of the nonlinearity function $y=f(x) ; 0 \leq x, y \leq 1$, it follows that the transmitter will pre-distort the signal $x(t)$ by using

$$
\hat{x}_{I}(t)=f^{-1}(x(t))
$$

Now, let's assume a discrete (sampled) system where the sampling period is $T_{s}$. Hence, we can write

$$
\begin{aligned}
\hat{x}_{I, n} & =\hat{x}_{I}\left(n T_{s}\right)=f^{-1}\left(x\left(n T_{s}\right)\right) \\
& =f^{-1}\left(x_{n-1}+\Delta x\right)
\end{aligned}
$$

where $x_{n-1}=x\left((n-1) T_{s}\right)$ and $\Delta(x)=x_{n}-x_{n-1}=T_{s}$.

Let's consider the simplest form of a predictor, i.e., a linear predictor which estimates the next non-linearity predistortion value, from the last two collected non-linearity points (i.e, $\left(x_{n-2}, f^{-1}\left(x_{n-2}\right)\right)$ and $\left.\left(x_{n-1}, f^{-1}\left(x_{n-1}\right)\right)\right)$, based on the following linear extrapolation

$$
\begin{aligned}
\hat{x}_{P, n} & =\hat{x}_{P}\left(n T_{s}\right) \\
& =\left[\left.\frac{d}{d x}\left(f^{-1}(x)\right)\right|_{x=x_{n-1}}\right] \cdot \Delta x+\left.f^{-1}(x)\right|_{x=x_{n-1}}
\end{aligned}
$$

In a practical implementation, $\left.\frac{d}{d x}\left(f^{-1}(x)\right)\right|_{x=x_{n-1}}$ can be approximated as

$$
\left.\frac{d}{d x}\left(f^{-1}(x)\right)\right|_{x=x_{n-1}}=\frac{f^{-1}\left(x_{n-1}\right)-f^{-1}\left(x_{n-2}\right)}{\Delta x}
$$

It is then straightforward to show that the percentage of error, that the above linear predictor exhibits, with respect to the ideal pre-distorter is computed as

$$
\begin{aligned}
e_{n} & =\frac{f\left(x_{n}=\hat{x}_{I, n}\right)-f\left(\hat{x}_{P, n}\right)}{f\left(x_{n}\right)} \\
& =\frac{f\left(x_{n-1}+\Delta x\right)-f\left(\left[\left.\frac{d}{d x}\left(f^{-1}(x)\right)\right|_{x=x_{n-1}}\right] \cdot \Delta x+\left.f^{-1}(x)\right|_{x=x_{n-1}}\right)}{f\left(x_{n-1}+\Delta x\right)}
\end{aligned}
$$

Or, we can write in a more generic form as

$$
\begin{aligned}
e(x) & =\frac{f(x)-f\left(\hat{x}_{P}\right)}{f(x)} \\
& =\frac{f(x+\Delta x)-f\left(\Delta x \cdot \frac{d}{d x}\left(f^{-1}(x)\right)+f^{-1}(x)\right)}{f(x+\Delta x)}
\end{aligned}
$$

Now let's examine some numerical results based on a typical power amplifier with a $3^{\text {rd }}$-order non-linearity. That is,

$$
f(x)=\frac{x+c \cdot x^{3}}{1+c} ; \frac{-1}{3} \leq c \leq 0
$$

It can be shown that the Total Harmonic Distortion (THD) for the above non-linearity (expressed in $\mathrm{dB}$ ) is calculated as

$$
\mathrm{THD}=20 * \log _{10}\left[\frac{-c}{(4+3 c)}\right] \mathrm{dB}
$$

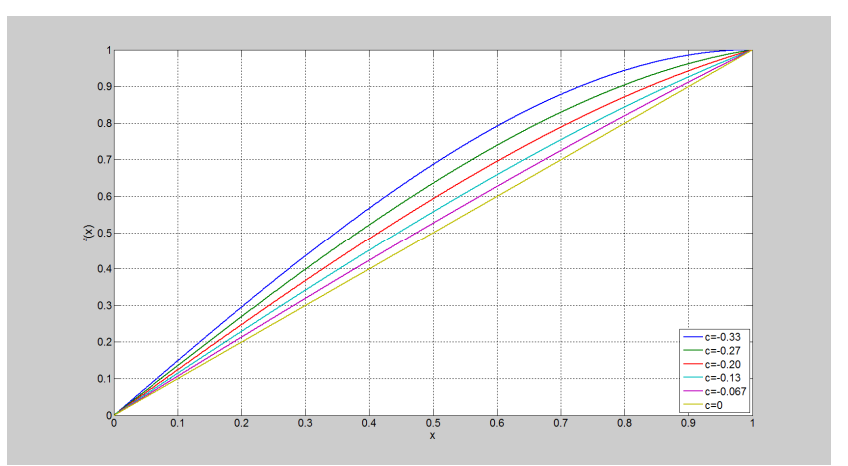

Figure 4. Plots of $f(x)$ for various " $c$ ". 
Figure 4 shows plots of $f(x)$ versus various levels of the 3rd order coefficient "c", while Figure 5 depicts a plot of the THD versus "c". Clearly, the worst-case non-linearity, for which it is expected to suffer the poorest linear predictor's performance, is when $c=-1 / 3$.

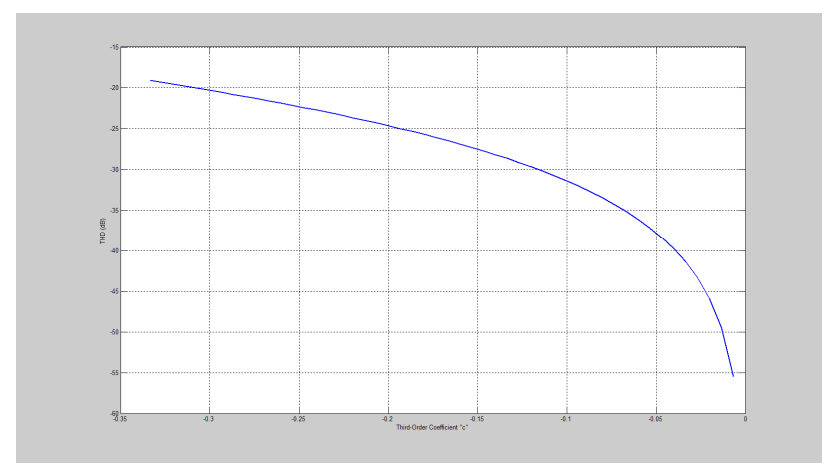

Figure 5. $T H D(d B)$ versus " $c$ ”.

Figure 6 to Figure 8 show plots of the error (\%) versus the prediction distance ${ }^{5} \Delta x$ and the non-linearity level (reflected by the value of " $c$ "). As expected, the smaller " $c$ ", the more prominent the non-linearity and the harder to predict the correct pre-distortion value. Also, the larger the prediction distance, $\Delta x$, the more inaccurate the slope prediction of $f^{-1}(x)$, hence, causing poorer prediction.

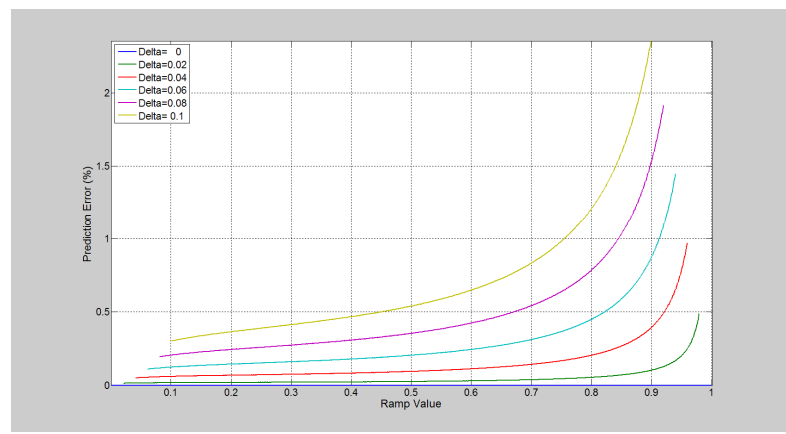

Figure 6. Prediction Error for a $3^{\text {rd }}$-Order Non-Linearity Device with $c=$ $-1 / 3$ [Ramp Value $=x]$.

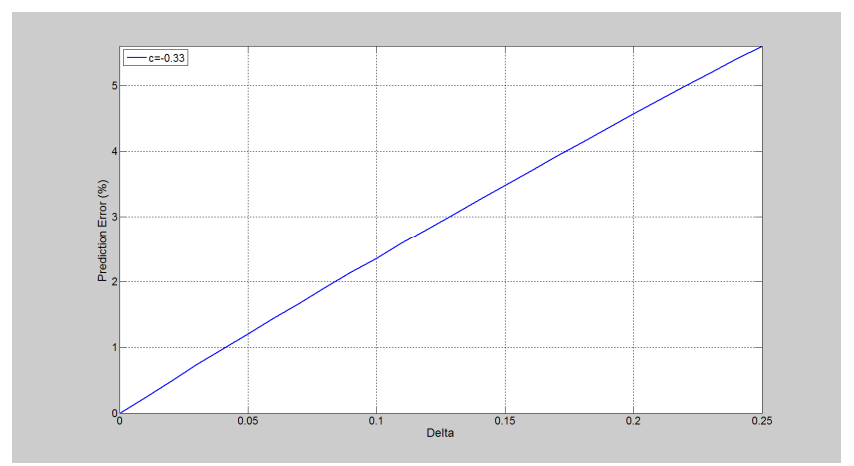

Figure 7. Maximum Prediction Error (over Ramp Value) for a $3^{\text {rd }}$-Order Non-Linearity Device with $c=-1 / 3[$ Delta $=\Delta x]$.

\footnotetext{
${ }^{5}$ Recall that the relationship between the sampling period, $T_{s}$, and the prediction distance, $\Delta x$, is $T_{s}=\Delta x$.
}

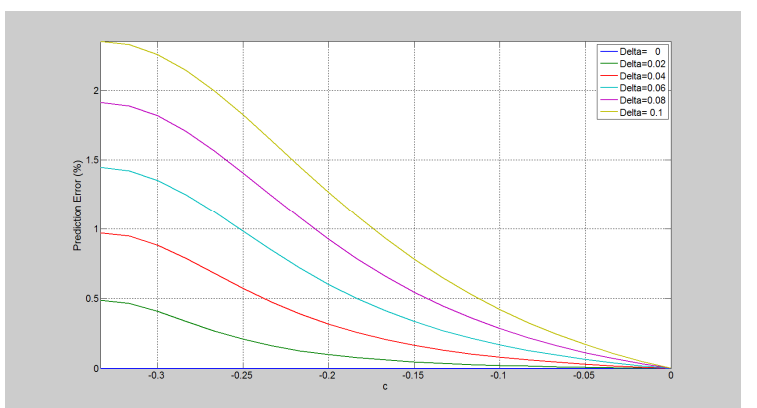

Figure 8. Maximum Prediction Error (over Ramp Value) for a $3^{\text {rd }}$-Order Non-Linearity Device with versus $\boldsymbol{c}$.

\section{Introduction to GSM/EDGE, UMTS Transmitter Performance Metrics}

The mobile transmitter must transmit enough power, with sufficient fidelity to maintain a call of acceptable signal quality, without transmitting excessive power into the frequency channels and timeslots allocated to others. In order to measure the performance of the transmitter, three zones of performance and emission measurements are deemed critical, namely, in-channel, out-of-channel and out-of-band emission. In the case of in-channel measurements, four quantities are typically used to characterize the user equipment (mobile handset) [2][3], namely, phase error, mean frequency error, mean transmitted RF carrier power, and transmitted $R F$ carrier power versus time. The out-of-channel measurements are carried out to determine how much interference the user causes to other GSM/EDGE, UMTS users such as spectrum due to modulation and wideband noise, spectrum due to switching, spurious emissions in the transmit and receive band [2][3]. Finally, out-of-band measurements determine how much interference the user causes other users of the radio spectrum across the wideband, especially into the receiver band [2][3].

\section{A. Modulation Accuracy Metrics}

In GSM/EDGE systems, the accuracy of modulation is determined by the phase error encountered for GMSK modulation (GSM) and the error vector magnitude (EVM) performance for 8-PSK modulation (EDGE). These tests can be considered as the fundamental parameters to measure the performance of the transmitter as outlined in the 3GPP standard [2][3]. Any impairment in the transmitter circuitry line-up may result in poor phase error or EVM performance. These parameters also determine the ability of a receiver to correctly demodulate, especially when the signal-to-noise ratio (SNR) is low. In general, RF frequency and phase errors are mainly due to impairments of the transmitter's phaselocked loop (PLL) and/or synthesizer circuitry generating the $\mathrm{RF}$ carrier. This could result in the target receiver not being able to synchronize properly to the transmitted bit stream. In addition, the transmitter might cause interference to adjacent channels, if the carrier frequency error is rather large.

B. Mean Transmitted RF Carrier Power

In GSM/EDGE systems [2][3], dynamic power control is typically used to ensure that each link maintains a certain 
minimum power requirement. If a transmitter produces too little power, link performance is compromised. On the other hand, if too much power is transmitted, the emitted signal will interfere with others and battery life is also reduced. The mean transmitted RF carrier power is defined as the mean power during the useful part of the GSM/EDGE burst [2][3].

C. Transmitted RF Carrier Power versus Time

This measurement is done to assess the envelope of the carrier power in the time domain against a prescribed time mask. In TDMA systems such as the GSM/EDGE system, transmitters must properly ramp the power up and down every burst in order to prevent adjacent timeslot interference. If transmitters turn on too slowly, data at the beginning of the burst might be lost, degrading link quality, and if they turn off too slowly the user of the next timeslot in the TDMA frame will experience interference.

D. Spectrum due to Modulation and Wideband Noise

Since the modulation process in a transmitter causes the continuous wave $(\mathrm{CW})$ carrier to spread spectrally, this measurement is designed/specified to ensure that modulation process does not cause excessive spectral-spread, which would result in interference in the other frequency bands. This measurement of spectrum due to modulation and wideband noise can be thought of as an adjacent channel power (ACP) measurement.

E. Spectrum due to Switching Transients

During the RF power ramp up and down in a TDMA (or a TDD) burst, undesirable spectral components can be introduced in the transmitted signal due to the switching effect. Therefore, this measurement is carried out to ensure that these un-desirable switching components are weaker than a certain minimum. If a transmitter ramps power too quickly, users operating on different frequencies, especially adjacent channel will experience significant interference.

F. Spurious Emissions

These measurements are done to make sure that transmitters do not put power into the wrong part of the spectrum. These modulated or un-modulated radiated or conducted spurious emissions can be in the form of transmit and receive band spurs which affect the system in an "inband" fashion, or they can also be cross-band and out-ofband spurious emissions, which would affect other systems in the vicinity of the operating transmitter.

\section{Performance Results}

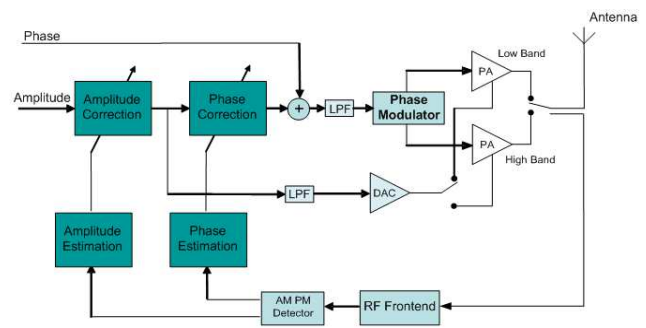

Figure 9. Transmitter/PA Block Diagram and Model with Feedback Circuit and Predictor Algorithm Digital Module.
Low Band
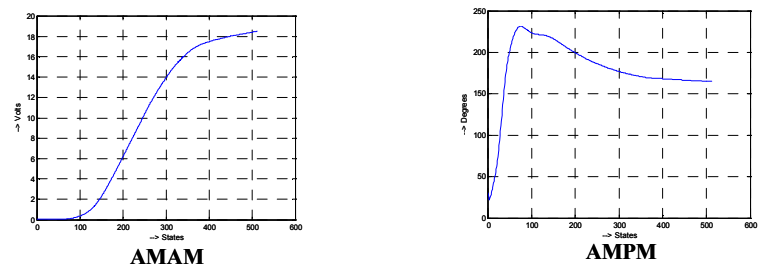

High Band
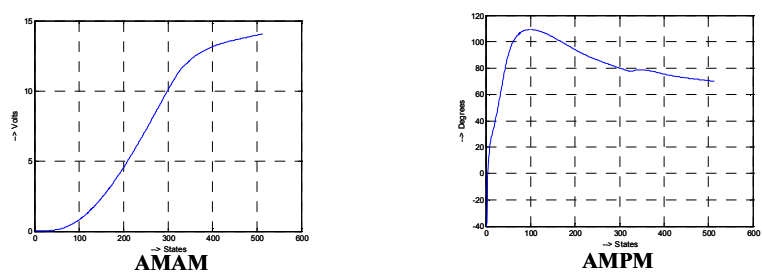

Figure 10. GSM PA Characteristics.

$\underline{\text { Low Band }}$

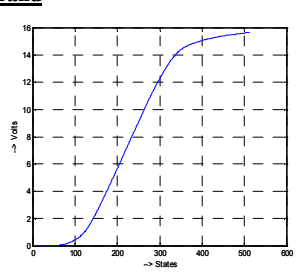

AMAM

High Band

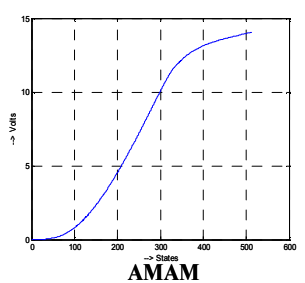

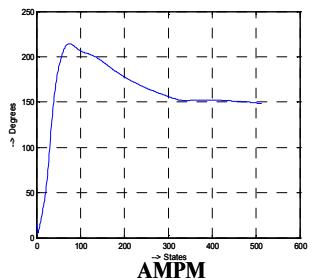

AMPM

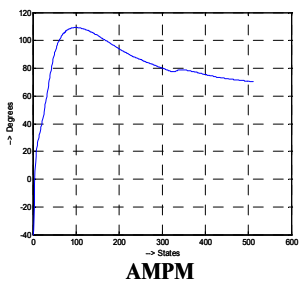

AMPM
Figure 11. EDGE PA Characteristics.

Figure 9, depicts a block diagram of our novel transmitter/PA predictive linearization system, The AM/AM (output amplitude vs. input amplitude) and the AM/PM (output phase vs. input amplitude) characteristics of the PA for both low band (GSM850 and GSM900) and high band (DCS1800 and PCS1900) for GSM and EDGE are shown in Figure 7 and Figure 8, respectively6. Further the AM/AM and $\mathrm{AM} / \mathrm{PM}$ characteristics shown in Figure 10 and Figure 11 are fitted data from the measured data of the amplifiers. The power amplifiers are highly non-linear as can be seen from the $\mathrm{AM} / \mathrm{AM}$ and $\mathrm{AM} / \mathrm{PM}$ characteristics. The improvement in the power spectrum with and without the proposed correction is shown in Figure 9 for a UMTS signal.

6 The PA characteristics, depicted in Figure 10 and Figure 11, are courtesy of M/A-COM Tyco Electronics. The reader is also referred to a version of the PA described in [8] for additional background. 


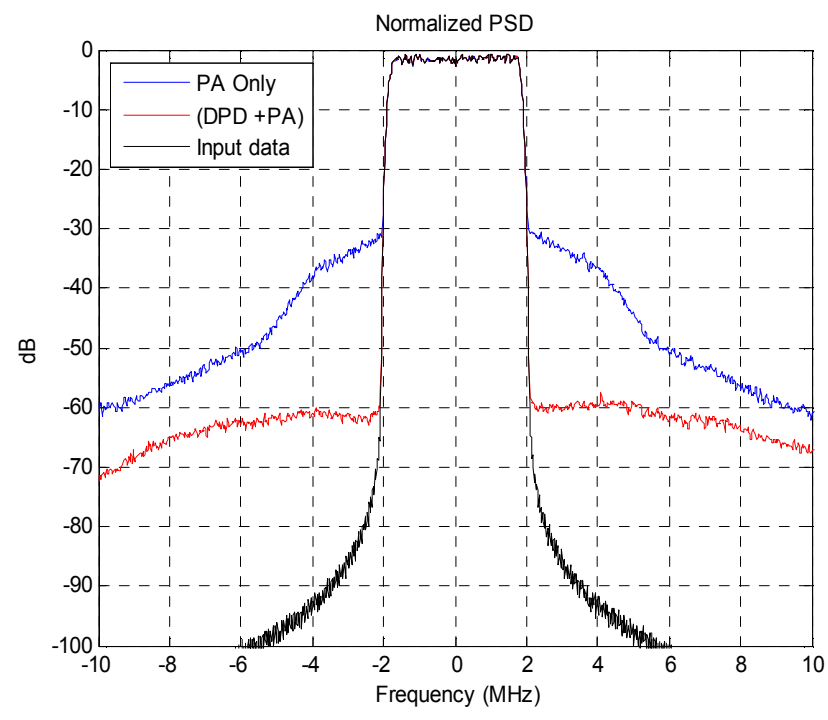

Figure 12. Spectral plot with and without digital pre-distortion.

Table 1. Detector Path Impairments.

\begin{tabular}{|c|c|c|c|c|}
\hline Parameter & Min. & Typ. & Max. & Unit \\
\hline SSB Phase Noise@1kHz & & -125 & & $\mathrm{dBc} / \mathrm{Hz}$ \\
\hline $\begin{array}{l}\text { Overall Feedback Path Noise } \\
\text { Figure }\end{array}$ & & 4 & 6 & dB \\
\hline Rx SNR & & 20 & & $\mathrm{~dB}$ \\
\hline IQ Gain Imbalance & & 1 & 3 & $\mathrm{~dB}$ \\
\hline IQ Phase Imbalance & & 1 & & degree \\
\hline Transmitter EVM & & 3 & 5 & $\% \mathrm{rms}$ \\
\hline ADC DNL/INL & & & 1 & bit \\
\hline
\end{tabular}

In our extensive simulations of the system shown in Figure 9, we have incorporated a significant amount of detail with regards to the modeling of the individual system components. Such models have been based on laboratory measurement results of the perspective components. Table 1, lists some of the key RF/Analog impairments that have been modeled into the simulations of the predictive loop.

For the numerical results provided in this section, the performance metrics we consider are switching transients (ST), average power and peak power at the PA and antenna outputs, the power-added-efficiency (PAE, in \%) at the PA and at the antenna outputs, adjacent channel power ratio (ACPR), the error vector magnitude (EVM) for EDGE and the RMS phase error for GSM. In order to compute the switching transients (see [2][3] for more details), the output signal from the output of the power amplifier is filtered with filters of $30 \mathrm{kHz}$ bandwidth centered at the following frequencies offset $400 \mathrm{kHz}, 600 \mathrm{kHz}$ and $1200 \mathrm{KHz}$, and of $100 \mathrm{kHz}$ bandwidth centered at $1800 \mathrm{kHz}$ offset. The 3GPP switching transient and ACPR specifications are summarized in Table 2.

Figure 10 shows the performance of our transmitter/PA system with the PA non-linearity left as is, i.e., uncorrected. That is, without the application of pre-distortion (e.g., pretabulated linearization) or the use of the predictive loop for an EDGE burst example. In the figure, the upper graph depicts the time-domain waveform of the EDGE burst at the PA output plotted versus the EDGE time-mask specification (in $\mathrm{dB}$ ). On the same plot, we also show the switching transient waveform performance versus time where the various waveforms represent the performance margin (in $\mathrm{dB}$ ) with respect to the EDGE ST specifications listed in Table 2. That is, the $\mathrm{dB}$ amount (versus time) shown on the figure (the red, blue and green graphs) represent the amount of $\mathrm{dB}$ violation of the resulting switching transient response/performance with respect to the EDGE standard specifications/requirement ${ }^{7}$. For example, in Figure 10 the uncorrected PA non-linearity during the ramp-up period is causing about $\sim 15 \mathrm{~dB}$ violation of the EDGE spec as seen in the figure by the red, blue and green graphs. Finally, the bottom graph in Figure 10 shows the modulation spectrum of the corresponding signals compared with the EDGE modulation spectral mask (the red curve). As mentioned earlier, the severe non-linearity of such the efficient PA used for our case-study significantly degrades the switching transient performance, especially during power ramp-up ( $\sim 15 \mathrm{~dB}$ violation of the EDGE specification!).

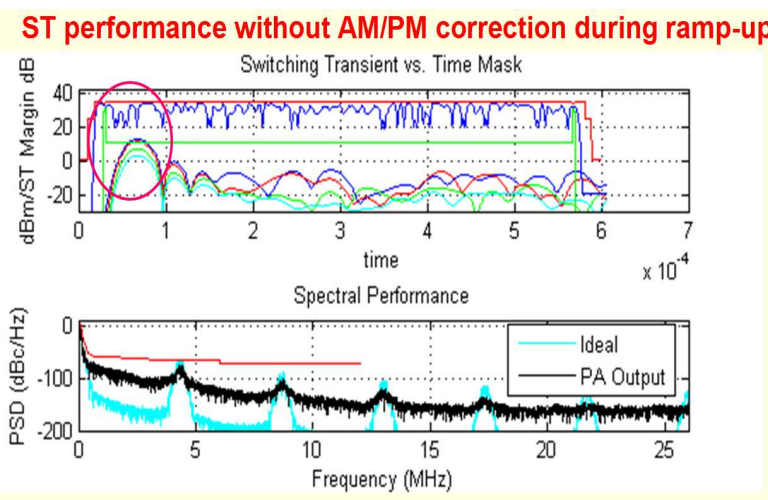

Figure 13. Switching Transient Performance of the Transmitter without Predictive Linearization on the EDGE Burst Ramp-up Interval.

Figure 14 to Figure 19 show a suite of performance results for GSM and EDGE modulation bursts with the application of our novel predictive linearization loop during the burst power ramp-up interval [8]. Also, Figure 20 shows examples of the time waveforms, during an EDGE modulation burst, at various stages inside the predictive loop.

As described earlier, upon completion of the burst ramp-up, all points on the PA non-linearity curve over the full range of PA gain bias would be completed and the pre-distortion linearization curve would be fully constructed. Hence, no need for prediction beyond the ramp-up time and direct predistortion using the constructed table can be used. In other words, the GSM/EDGE ramp-up duration is used in our predictive approach as the "training" period to collect the PA pre-distortion table and during such training period, prediction is used to compensate for the lack of the pre-

\footnotetext{
${ }^{7}$ Hence, a positive deviation (i.e., the graph is above the $0 \mathrm{~dB}$ line) means a violation, while a negative deviation (i.e., the graph is below the $0 \mathrm{~dB}$ ) line means a margin.

8 Note that for the GSM case, the peak and average power curves are the same and therefore, the blue curves coincide on the red curves and hence only the blue curve is visible.
} 
distortion table, which is still being collected.

Table 2. Switching Transients and ACPR Specifications (3GPP).

\begin{tabular}{|c|c|c|c|c|}
\hline \multirow{2}{*}{ Center Frequency (kHz) } & \multirow{2}{*}{ Bandwidth (kHz) } & \multicolumn{2}{|c|}{ ACPR Limit (dBc) } & \multirow{2}{*}{ ST Limit (dBm) } \\
\hline & & GSM & EDGE & \\
\hline 400 & 30 & -60 & -54 & -23 \\
\hline 600 & 30 & -60 & -60 & -26 \\
\hline 1200 & 30 & -60 & -60 & -32 \\
\hline 1800 & 100 & -63 & -63 & -36 \\
\hline
\end{tabular}
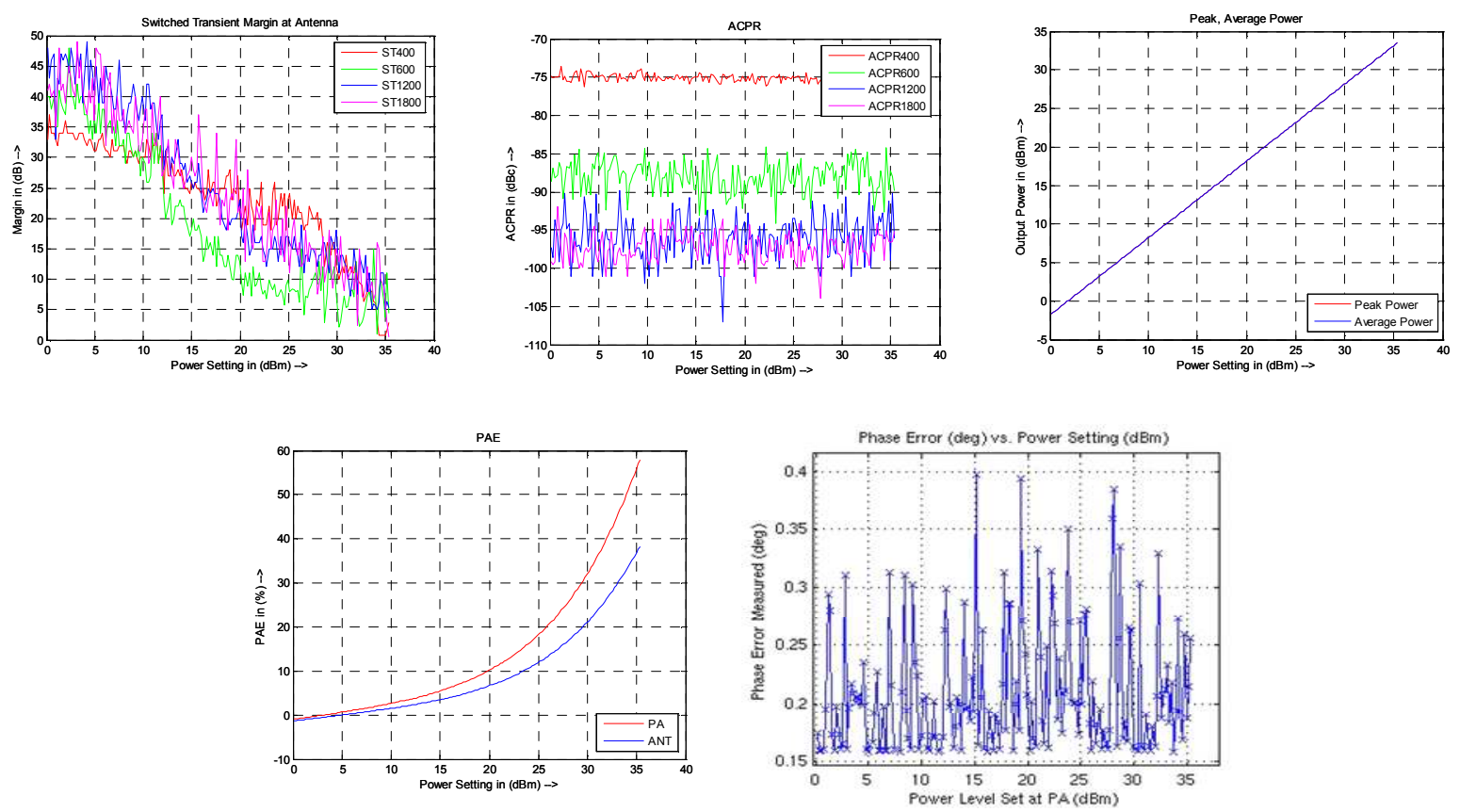

Figure 14. GSM Low Band Performance Metrics.
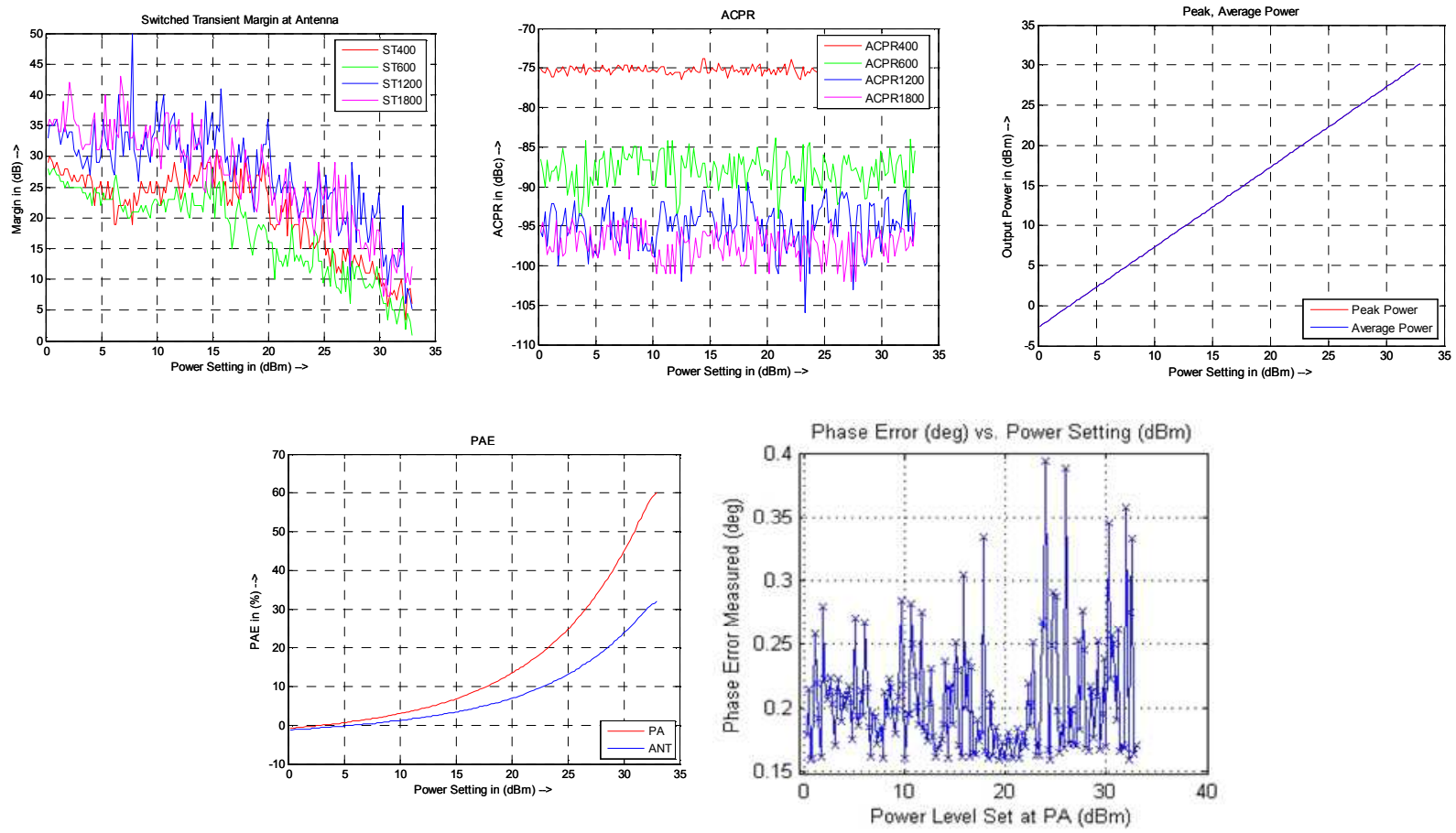

Figure 15. GSM High Band Performance Metrics. 

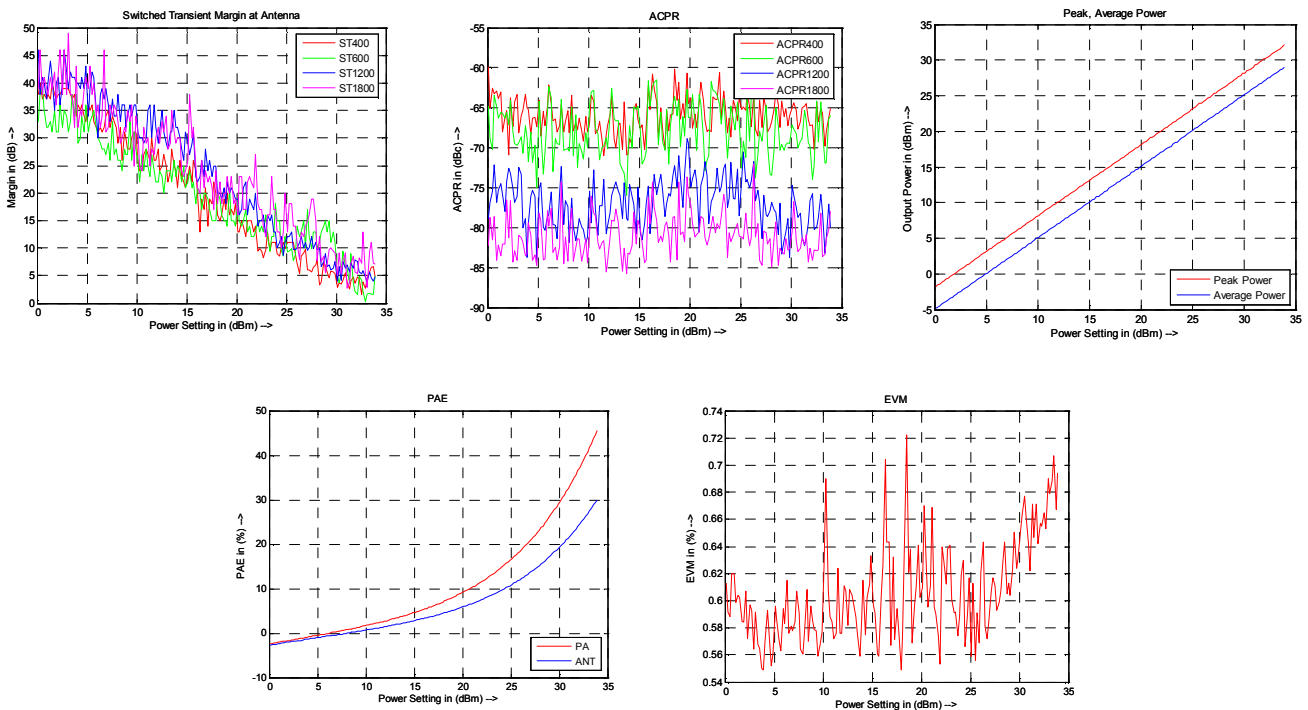

Figure 16. EDGE Low Band Performance Metrics.
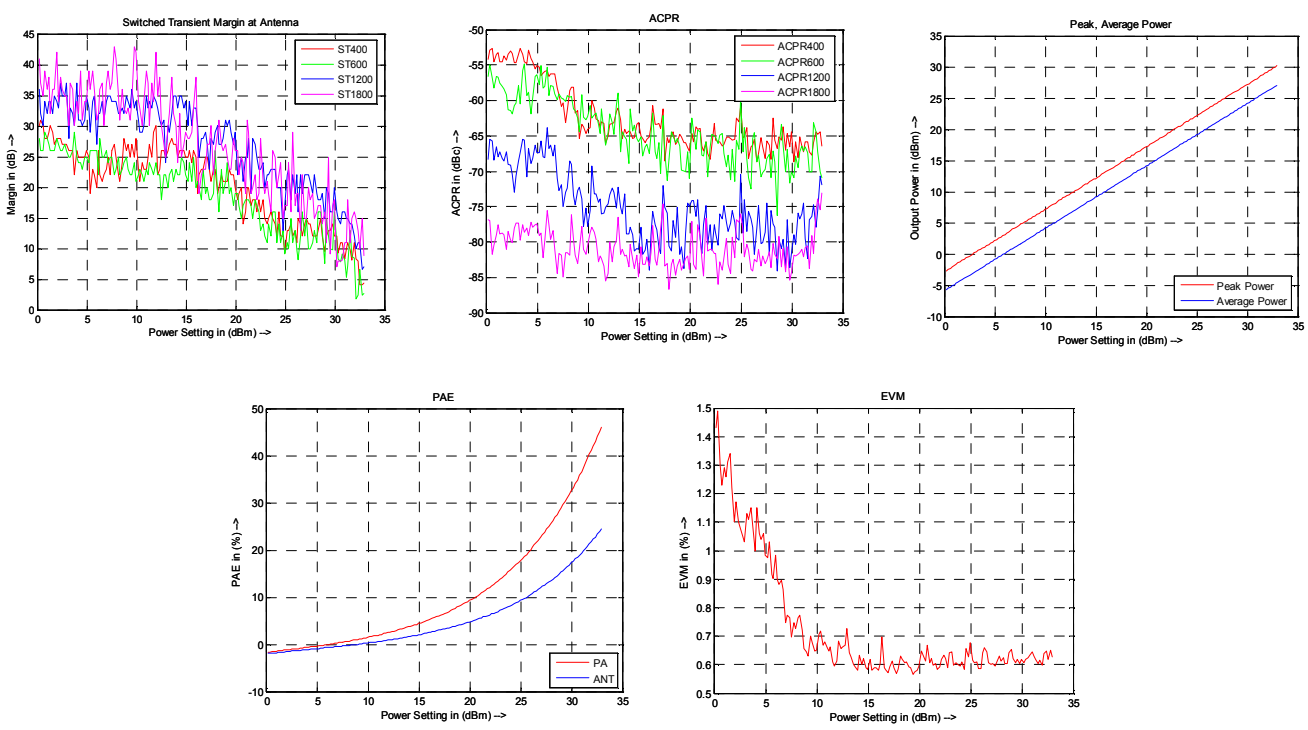

Figure 17. EDGE High Band Performance Metrics.

Switching Transient vs. Time Mask
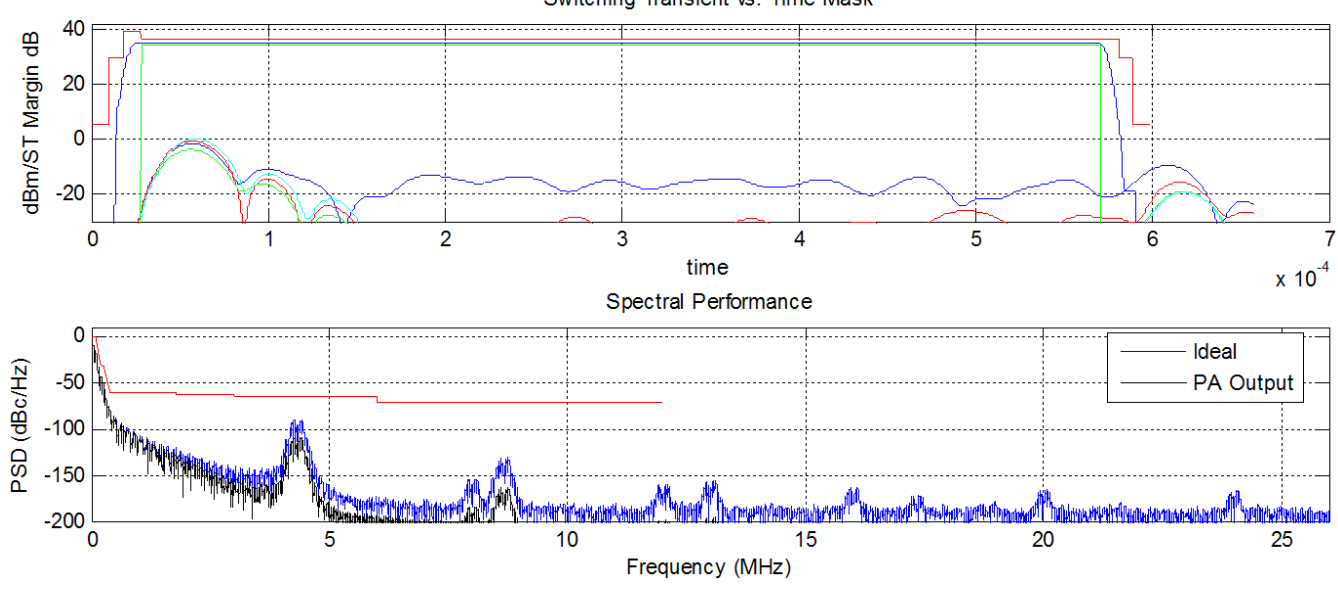

Figure 18. GSM Switching Transients and Spectral Performance Plots. 

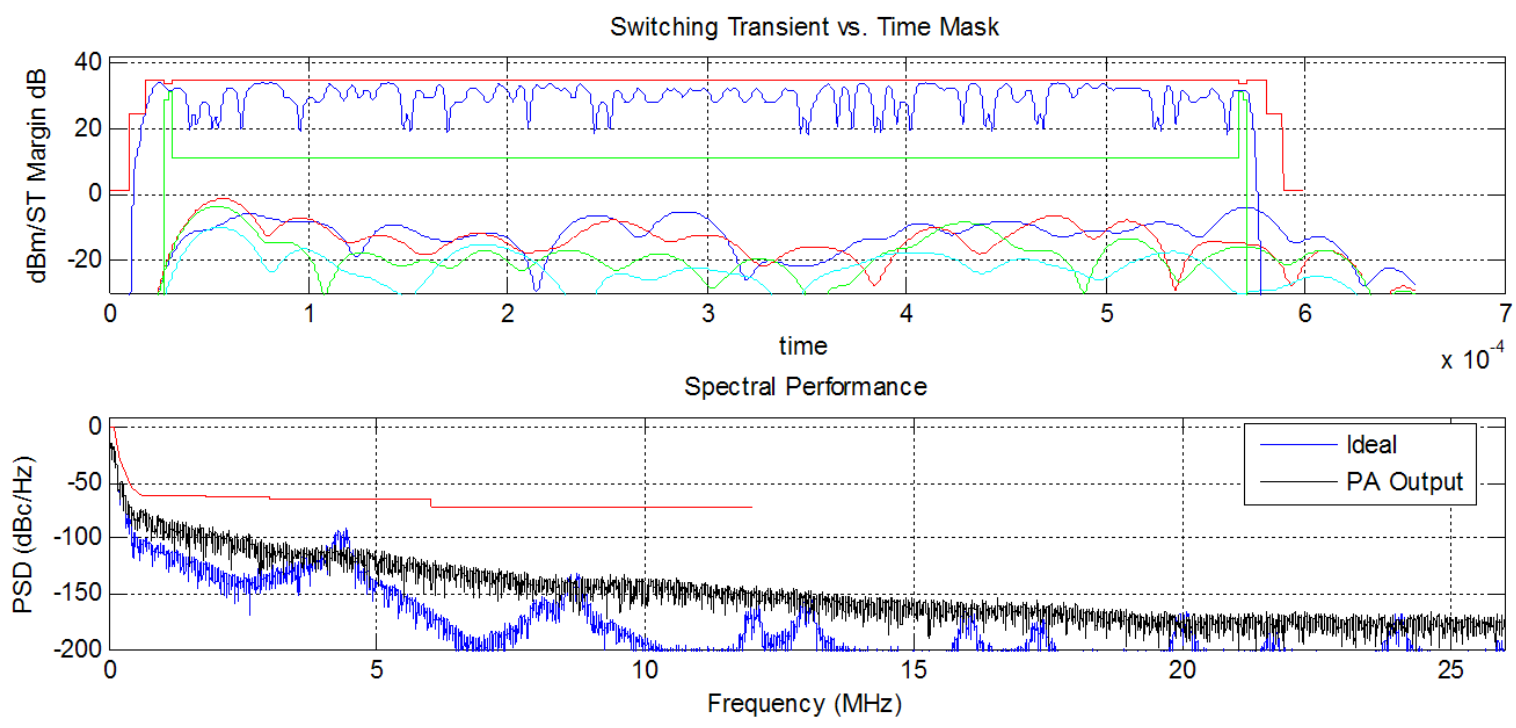

Figure 19. EDGE Switching Transients and Spectral Performance Plots.

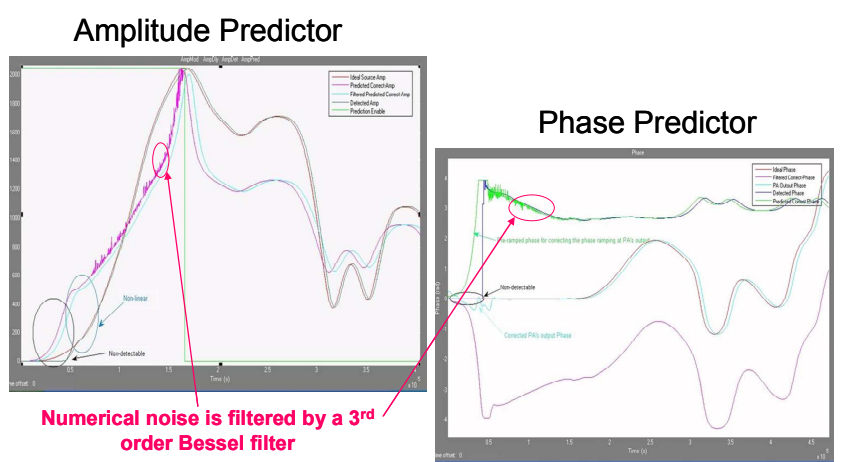

Figure 20. Examples of Waveforms at Various Stages within the Predictive Loop.

It should be noted that in our transmitter system (and for the results in Figure 14 to Figure 19), the maximum (peak) power for low-band has been designed, or set at $35.35 \mathrm{dBm}$, while for the high-band it has been set at $32.9 \mathrm{dBm}$. Also, for the results presented in this section, the assumed insertion loss after the PA for GSM/EDGE high-band has been $2.75 \mathrm{~dB}$ and for GSM/EDGE low-band it has been $1.81 \mathrm{~dB}$, to reflect the PA insertion loss at the time of obtaining those results. The EVM has been measured at the transmitter's output, while the PAE has been calculated based on PA measurements for various bias points. Finally, the Rx in-band noise has been measured at both the PA output and at the output of the antenna (with the insertion loss included).

As can be seen from all the performance results presented in Figure 14 to Figure 19, the predictive loop has helped to successfully eliminate the large switching transient violations encountered in the no-correction case (as in Figure 13) without the need to use pre-stored linearization (predistortion) tables. As shown in Figure 18, at the peak output level of $33.5 \mathrm{dBm}$ at antenna, the Switching Transient performance has improved significantly to enjoy a minimum of about $2 \mathrm{~dB}$ of margin at the antenna for GSM. Also, in Figure 19 , there is more than $3 \mathrm{~dB}$ performance margin at the peak output level of $32.1 \mathrm{dBm}$ at antenna for EDGE. Overall, the $\mathrm{AM} / \mathrm{AM}$ and $\mathrm{AM} / \mathrm{PM}$ correction has improved the performance with about $3 \sim 6 \mathrm{~dB}$, as compared to the nocorrection (i.e., without the predictive loop) case.

As mentioned earlier, the spectral plots for the proposed method with a UMTS signal shown in Figure 12, demonstrate the improvement of the spectral re-growth for the transmitted signal with our technique over the case without pre-distorting the signal. Comparing the power spectral density (PSD) and the adjacent channel power ratio (ACPR) the performance of the pre-distorter clearly shows that the signal without the proposed pre-distortion regenerates out of band spectrum, while with the proposed pre-distortion can effectively suppress the regeneration. It can be seen that the PSD and ACPR is improved in comparison to the transmitted signal.

Wider bandwidths are further possible but there is a need for the change in the structure of the architecture and the LUT's which is beyond the scope of discussion in this paper.

\section{Conclusion}

In this paper, we presented a novel real-time closed-loop device linearization technique that is based on on-the-fly prediction of the non-linear characteristics of interest. We have applied our novel predictive "pre-distorter" technique to the challenging problem of maintaining linearity of highly efficient (thus highly non-linear) $2.5 \mathrm{G}$ transmitters during power ramp-up of a GSM/EDGE burst in order to meet the stringent performance requirements of such systems, such as switching transients, with minimal factory pre-calibration of the transmitter and/or the power amplifier to acquire the necessary pre-distortion tables. We have also provided a simplified, rather generic, mathematical frame-work for the expected performance of the predictive approach and conducted extensive simulations based on detailed modeling of our transmitter and PA design and laboratory 
measurements. The results of our analysis have demonstrated that our novel predictor-based closed-loop approach used for calibration of the power amplifier is able to linearize the PA during the power ramp-up duration in a GSM/EDGE burst, without any prior calibration of the PA. Such is a very promising result compared to existing solutions as it overcomes the natural (causal) delay encountered with any on-the-fly feedback loop calibration technique; a problem that has a degrading effect of the real-time calibration loop and has not been solved in prior work. The simplicity if our approach and its ability to operate with minimal or no precalibration data qualifies it as a strong candidate for on-thefly calibration/linearization of highly-efficient non-linear power amplifiers regardless of the variations due to temperature, process, power and aging. Hence, it presents a promising solution for mobile handset transmitter design.

\section{References}

[1] Walid K. M. Ahmed and Qing Li, "Method and Apparatus for a Nonlinear Feedback Control System". U.S. Patent\# $7,889,810$.

[2] 3GPP TS 45.005 V7.8.0. Digital Cellular Telecommunications Systems; Radio transmission and reception (Release 2006-11).

[3] 3GPP TS 51.021 V7.1.0. Base Station System (BSS) equipment specification; Radio aspects (Release 2005-11).

[4] Walid Ahmed, "Methods and Apparatus for Signal Power Ramp-up in a Communication System", U.S. Patent Application No. 11/385212.

[5] M. Nezami, "Fundamentals of Power Amplifier Linearization Using Digital Pre-Distortion", High Frequency Electronics, September 2004, pp. 54-59.

[6] W. Ahmed and D. Douglas, "Multi-mode selectable modulation architecture calibration and power control apparatus, system, and method for radio frequency power amplifier". U.S. Patent\# 7,599,448.
[7] SCHETZEN M.: "The Volterra and Wiener theories of nonlinear systems' (Krieger Publishing Co., 2006).

[8] P. Nagle, R. Husseini, A. Grebennikov, W. K. M. Ahmed and F. McGrath, "A novel wideband digital power amplifier and transmitter architecture for multimode handsets", In Proc. Of the 2004 IEEE Radio and Wireless Conference, pp. 171 - 174.

[9] Walid K. M. Ahmed, "Quantization Noise Suppression in Digitally Segmented Amplifiers", IEEE Transactions on Circuits and Systems I: Regular Papers, Volume 56, Issue 3, March 2009, pp. 529 - 540.

[10] P. L. Gilabert, A. Cesari, G. Montoro, E. Bertran, and J.-M. Dilhac, "Multi-lookup table FPGA implementation of an adaptive digital predistorter for linearizing RF power amplifiers with memory effects," IEEE Trans. Microw. Theory Tech., vol. 56, no. 2, pp. 372-384, Feb. 2008.

[11] A. Zhu, P. J. Draxler, J. J. Yan, T. J. Brazil, D. F. Kimball, P. M. Asbeck, " Open-Loop Digital Predistorter for RF Power Amplifiers Using Dynamic Deviation Reduction-Based Volterra Series," IEEE Trans. Microw. Theory Tech., vol. 56, no. 7 , pp. 1524-1534, July 2008.

[12] S.Saied-Bouajina, O. Hammi, M.Jaidane-Saidane and F.M. Ghannouchi, "Experimental approach for robust identification of radiofrequency power amplifier amplifier behavior models using polynomial structures", IET Microwave Antennas Propogation, 2010, vol. 4, issue 11, pp. 1818-1828.

[13] Ding L., Zhou G.T., Morgan D.R., et al. 'A robust digital baseband predistorter constructed using memory polynomials', IEEE Trans. Commun., 2004, 52, (1), pp. 159-165.

[14] Kim J., Konstantinou K.: 'Digital predistortion of wideband signals based on power amplifier model with memory', Electron. Lett., 2001, 37, (23), pp. 1417-1418.

[15] Jeckeln E.G., Beauregard F., Sawan M.A., Ghannouchi F.M.'Adaptive baseband/RF predistorter for power amplifiers through instantaneous AM-AM and AM-PM characterization using digital receivers'. Dieg. 2000 IEEE MTT-S Int. Microwave Symp., Boston, MA, USA, June 2000, pp. 489492. 